\title{
Construção de Objetos de Aprendizagem a partir de um software de Geometria Dinâmica: uma proposta de capacitação para professores de Matemática
}

\author{
Bárbara Gorziza Ávila - barbaragorzizaavila@gmail.com - PPGIE - UFRGS \\ Thaísa Jacintho Müller - thaisa.muller@ pucrs.br - PPGIE - UFRGS - PUCRS \\ Liane Margarida Rockenbach Tarouco - liane@ penta.ufrgs.br - PPGIE - UFRGS \\ José Valdeni de Lima - valdeni@inf.ufrgs.br - PPGIE - UFRGS
}

\begin{abstract}
Resumo: Este artigo apresenta o relato de uma experiência realizada com professores e futuros professores de Matemática com objetivo de promover uma capacitação para o uso de recursos tecnológicos em sala de aula, mais especificamente construção de Objetos de Aprendizagem utilizando o software GeoGebra. Ancorada nas ideias da Aprendizagem Complexa e nos conceitos e estudos já existentes sobre produção e utilização de Objetos de Aprendizagem, tal proposta realizou-se em forma de curso de extensão, na modalidade semipresencial, em que os participantes tiveram contato com a ferramenta e apresentaram produções concretas a serem utilizadas com seus alunos, o que certamente virá a contribuir na promoção da aprendizagem matemática destes estudantes.
\end{abstract}

Palavras Chave - aprendizagem complexa, GeoGebra, objetos de aprendizagem

\begin{abstract}
This paper presents a report conducted with present and future mathematics teachers in order to promote training about the use of technological resources in classroom, specifically focusing on construction of Learning Objects using GeoGebra software. Anchored in the concept of Complex Learning and existing studies on learning objects production and use, this proposal was held in the form of an extension course in hybrid format, in which participants had contact with the tool and presented productions to be used with their students, what will certainly contribute to the promotion of mathematics learning of these students.
\end{abstract}

Keywords - Complex Learning, GeoGebra, Learning Objects

\section{Introdução}

O sistema educacional brasileiro tem revelado uma série de deficiências que vêm comprometendo o desempenho dos seus estudantes com relação à performance alcançada em países considerados de primeiro mundo. Dados fornecidos pelo Instituto Nacional de Estudos e Pesquisas INEP (2013) apontam que, de acordo com as metas traçadas pelo Termo de Adesão ao Compromisso "Todos pela Educação", o Brasil só deverá se aproximar do nível de qualidade do sistema educacional dos países que constituem a Organização para a Cooperação e Desenvolvimento Econômico (OCDE) ${ }^{1}$ em meados de 2021. Além disso, dados extraídos do SAEB (2011) evidenciam que o estudante brasileiro da educação básica tem apresentado um nível de proficiência mínimo na disciplina de Matemática.

\footnotetext{
${ }^{1}$ Maiores informações: http://www.oecd.org/fr/
} 
Neste cenário, o aprimoramento docente deve ser considerado um elemento essencial, visto que o desempenho do aluno está diretamente relacionado à qualidade da prática pedagógica oferecida pelo professor (CHETTY, FRIEDMAN e ROCKOFF, 2013). Com base nesta perspectiva, pesquisas na área da educação Matemática, tais como as desenvolvidas por Devlin (2012); Heng e Sudarshan (2013); DeCaro e Rittle Johnson (2012) e Aguirre et al. (2012) vêm se apoiando em novas estratégias pedagógicas, pautadas no processo de construção do conhecimento Matemático, em lugar do foco sobre resultados pontuais apresentados pelos estudantes.

As Tecnologias da Informação e Comunicação (TICs), com suas potencialidades em termos de interatividade e uso da multimídia, podem contribuir para o desenvolvimento de materiais educacionais que venham a atender as novas demandas pedagógicas. Para tanto, faz-se necessária a formação sobre ferramentas de autoria que possibilitem ao docente a elaboração de seus próprios materiais educacionais, de modo que estes sejam adaptados às suas necessidades e aspirações. Neste contexto, o presente artigo apresenta uma iniciativa de formação de docentes e licenciandos em Matemática sobre o uso da ferramenta GeoGebra, com vistas a promover a autoria sobre Objetos de Aprendizagem (OAs) a serem explorados com alunos da Educação Básica.

A pesquisa desenvolvida traz como fundamentação teórica os conceitos de Aprendizagem Complexa e Objetos de Aprendizagem, descritos nas seções 2 e 3 deste artigo. A seção 4 apresenta a metodologia desenvolvida na pesquisa, seguida pelos resultados obtidos, descritos na seção 5. Por fim, são discutidas algumas considerações com relação ao trabalho desenvolvido.

\section{A Aprendizagem Complexa no âmbito da Matemática}

Uma mudança de paradigma vem se revelando nas pesquisas que envolvem o ensino de Matemática, alterando significativamente as concepções docentes sobre como a aprendizagem é construída pelo aluno. As novas ideias que devem permear o delineamento das práticas pedagógicas em Matemática já não comportam o mero reconhecimento de técnicas procedimentais, desconexas da realidade, a serem aplicadas sem a necessidade de uma reflexão conceitual. Para Devlin (2012) o novo desafio do professor de Matemática está na realização de ações pedagógicas que conduzam o estudante à construção de um Pensamento Matemático, o qual perpassa o reconhecimento de fórmulas e técnicas para a resolução de problemas, contemplando também a busca pela real compreensão dos conceitos matemáticos inerentes aos objetos estudados.

A compreensão de um conceito requer a capacidade de transferir suas aplicações para diferentes situações vivenciadas na prática diária. Para ensejar o desenvolvimento de tais habilidades, Van Merriënboer e Kirshner (2013) desenvolveram o conceito de Aprendizagem Complexa, segundo o qual a aprendizagem deve ser construída a partir da integração de três dimensões: habilidades, atitudes e conhecimento.

As habilidades devem prover um suporte para a aplicação prática dos conceitos inerentes ao conteúdo curricular. $\mathrm{O}$ domínio sobre softwares para a análise qualitativa, por exemplo, é uma habilidade técnica necessária à formação de profissionais da Estatística. Da mesma forma, o domínio sobre softwares de manipulação de objetos geométricos pode ajudar professores em formação no desenvolvimento de seus próprios Objetos de Aprendizagem, ou seja, trata-se de uma habilidade importante à prática docente. Com relação às atitudes, Van Merriëmboer e Kirshner (2013) enfatizam que a educação deve promover o desenvolvimento de uma postura adequada ao futuro profissional que o estudante deverá se tornar. Neste sentido, o engajamento em 
atividades colaborativas e cooperativas pode ser uma boa estratégia para se promover a capacidade de socialização e trabalho em equipe. $\mathrm{O}$ conhecimento, mais especificamente no contexto da Matemática, está diretamente relacionado ao conceito de Pensamento Matemático, trazido por Devlin (2012). Se o estudante compreende os conceitos inerentes ao objeto de estudo, este torna-se apto não somente a replicar o conteúdo apresentado pelo professor em sala de aula, mas também a explorar seus conceitos em situações inusitadas que requeiram sua interpretação, com a identificação autônoma de aspectos relevantes a serem considerados nos problemas vivenciados.

Segundo Heng e Sudarshan (2013) e Aguirre et al. (2012), a capacidade de abstração humana tende a ser ampliada na medida em que se consolida uma fluência sobre o uso cotidiano da linguagem Matemática. Ou seja, a constante prática do pensamento Matemático conduz o estudante ao aprimoramento de suas habilidades de abstração sobre problemas cotidianos e de sua transcrição para a Linguagem Matemática. Neste sentido, o professor deve estimular a leitura e interpretação de problemas, relacionando a linguagem Matemática com informações já conhecidas pelo estudante. Objetos de Aprendizagem para o ensino de Funções baseados na manipulação de parâmetros e gráficos podem estabelecer conexões entre problemas expressos via notação Matemática e suas implicações práticas, representadas através de gráficos. Por exemplo, o seguinte problema pode ser interpretado pelo aluno algebricamente e representado via gráfico, mostrando o comportamento da função (Figura 1): $O$ número de postagens dos alunos na comunidade do facebook da escola Vou Passar no Enem tem dobrado a cada ano desde que liberaram o acesso ao Facebook no laboratório de informática. Tendo em vista a forte relação de dependência entre conceitos Matemáticos, a aprendizagem daqueles que constituem pré-requisitos faz-se essencial para as novas aprendizagens. Heng e Sudarshan (2013) advertem que a existência de lacunas na compreensão de conceitos anteriores pode comprometer demasiadamente as novas aprendizagens. Dessa forma, um conceito mal compreendido pode prejudicar a apropriação de novos

conceitos que seriam ancorados ao mesmo, o que muitas vezes vem a ocasionar a falta de motivação e o

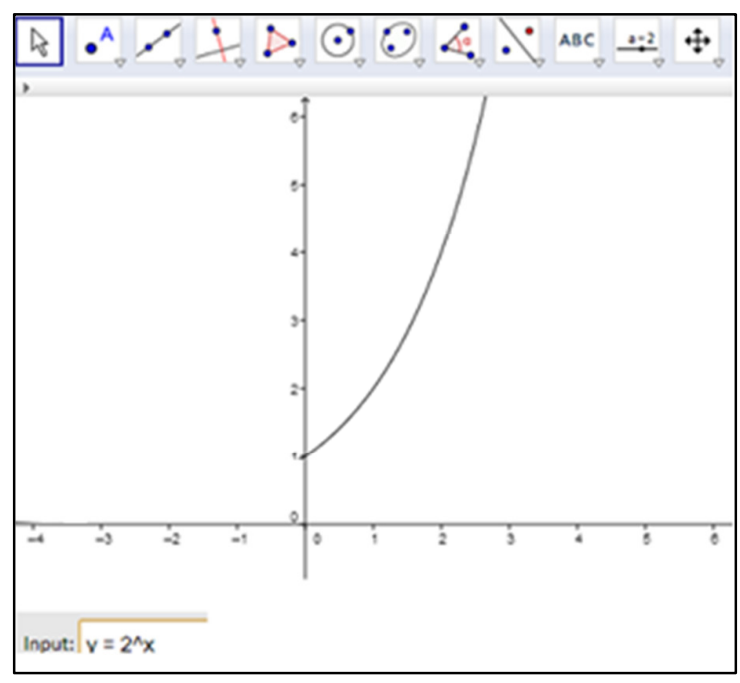

Figura 1- Representação algébrica e gráfico da função desinteresse do estudante pela Matemática. Pesquisas conduzidas por estas autoras concluem que os estudantes podem chegar a resultados corretos na resolução de problemas matemáticos atendo-se somente ao passo a passo dos procedimentos realizados pelo docente na resolução de problemas similares, sem uma real compreensão do todo. Ou seja, em muitas ocasiões, o aluno aparenta ter se apropriado do conhecimento, realizando com sucesso suas atividades, mas, no entanto, o que houve foi apenas a memorização dos procedimentos necessários para se chegar a um fim específico. 
DeCaro e Rittle Johnson (2012) advertem que este tipo de situação costuma ocorrer em função de que as práticas de ensino priorizam a apresentação do conteúdo com uma identificação prévia de todos os elementos-chave necessários para o alcance dos objetivos de aprendizagem. No primeiro momento aluno já se depara com todas as informações necessárias para a resolução dos problemas propostos, não havendo espaço para reflexão.

Para que ocorra a transposição desta prática pedagógica, que não enfatiza a análise e reflexão sobre os problemas Matemáticos, DeCaro e Rittle-Johnson (2012) sugerem a adoção de situações-problema. Segundo os autores, a formalização do conteúdo deve ser precedida por um engajamento investigativo por parte do aluno. $\mathrm{Ou}$ seja, a compreensão conceitual deve preceder a formalização dos conceitos matemáticos.

Neste contexto, Charlesworth e Leali (2012) apontam para quatro elementos considerados essenciais em atividades baseadas na resolução de problemas:

- Comunicação: As situações de ensino e aprendizagem devem oportunizar a troca de conhecimento entre os estudantes. Os problemas propostos devem ensejar a ocorrência de diálogos entre os envolvidos, de modo que a análise e reflexão sobre os aspectos importantes a serem considerados para a sua resolução devem ser realizadas de forma cooperativa entre o grupo.

- Estabelecimento de conexões: Problemas matemáticos devem ser relacionados à realidade cotidiana do aluno. Para que a aprendizagem faça sentido, é importante que o objeto de conhecimento seja contextualizado de modo que o aluno consiga identificar a relevância de sua aplicação nas situações cotidianas.

- Representação: A tradução dos problemas para a linguagem matemática deve ser objeto de constante reflexão. $\mathrm{O}$ aluno deve se utilizar da linguagem matemática com a mesma destreza que ele se utiliza de outras linguagens. A comunicação matemática é um elemento essencial para a construção do raciocínio abstrato.

- Raciocínio: O aluno deve ser constantemente estimulado a se engajar cognitivamente na busca pela compreensão dos conceitos envolvidos na situaçãoproblema. Muito mais importante do que a mera memorização dos procedimentos a serem adotados é a capacidade de leitura do problema com identificação de seus principais aspectos e reflexão sobre possíveis abordagens para a resolução do mesmo.

Esta breve abordagem teórica apresenta alguns, dentre os tantos aspectos, para os quais o docente deve atentar quando este se encontra envolvido na elaboração de Objetos de Aprendizagem (OAs) para o ensino da Matemática. Para dar segmento à discussão sobre proposta de construção e aplicações de Objetos de Aprendizagem nesta área de conhecimento, a próxima seção dedicar-se-á a uma abordagem sobre o conceito de OAs e a um estado da arte sobre propostas de construção e aplicação dos mesmos no contexto do ensino de Matemática.

\section{Objetos de Aprendizagem e suas aplicações}

A difusão das novas tecnologias no contexto educacional vem possibilitando o enriquecimento de materiais didáticos, que hoje tendem a assumir um caráter mais interativo e multimidiático. Além da possibilidade de elaboração de produtos mais efetivos, voltados para situações de ensino e aprendizagem pautadas numa postura ativa do estudante, o advento da internet veio a possibilitar a propagação e compartilhamento de recursos desenvolvidos pelos docentes.

As oportunidades de autoria e compartilhamento de recursos pedagógicos através da rede deram ensejo ao surgimento do termo Objetos de Aprendizagem, 
definido por Wiley (2000) como "[...] qualquer recurso digital que pode ser reusado para apoiar a aprendizagem". Incluem-se no rol dos Objetos de Aprendizagem quaisquer conteúdos digitais quando estes são explorados em um contexto educacional. Desse modo, podem ser citados como exemplos de Objetos de Aprendizagem conteúdos digitais envolvendo texto, imagem, áudio (ou a reunião de várias mídias), softwares educacionais, ou mesmo outros tipos de software, quando estes são explorados com fins pedagógicos.

Um aspecto de destaque contemplado pela definição de Wiley (2000) é o atributo da reusabilidade inerente a estes objetos. $\mathrm{O}$ conceito de reusabilidade abarca as múltiplas possibilidades de adaptação de um mesmo conteúdo a diferentes unidades de aprendizagem. Segundo Wiley (2000, p. 2) "Designers instrucionais podem construir pequenos (relativo ao escopo de um curso) componentes instrucionais que podem ser reusados várias vezes em diferentes contextos".

A elaboração de Objetos de Aprendizagem vem sendo facilitada pelo acesso a ferramentas de autoria que já não exigem do docente um amplo conhecimento em áreas como o design ou programação. No âmbito da Matemática, softwares que permitem a manipulação de elementos geométricos vêm se mostrando ferramentas promissoras para o desenvolvimento de atividades pedagógicas calcadas na experimentação e análise, proporcionando assim a ruptura de um modelo de ensino baseado meramente em ações procedimentais para um modelo que passa a dar ênfase na constituição do Pensamento Matemático.

Dentro desta perspectiva mais construtivista, Tabaghi e Sinclair (2013) conduziram uma investigação sobre o desenvolvimento do pensamento matemático de estudantes na medida em que estes interagiam com um software de Geometria Dinâmica para explorar os conceitos de autovalores e autovetores, essenciais para a compreensão da Álgebra Linear. Segundo os autores, a interação dos estudantes com esta ferramenta proporcionou o desenvolvimento de uma sintetização dos conceitos geométricos, favorecendo, em consequência, a aprendizagem dos conceitos inerentes à Algebra Linear. Roffkamp (2011) desenvolveu, com o apoio do software de Geometria Dinâmica Cinderella ${ }^{2}$, um conjunto de atividades baseadas em visualizações interativas com o intuito de auxiliar estudantes com idade de 15 a 16 anos na construção de conceitos e relações estabelecidos no ensino de funções, os quais fornecem a base para a aprendizagem do Cálculo. Os resultados da pesquisa mostraram que: “[...] as visualizações interativas não somente conduzem à manipulação de alguns pontos ou linhas, mas realmente ativam a formação de um acesso intuitivo a conceitos do Cálculo" (ROFFKAMP, 2011, p. 370). Ainda, Brandão, Isotani e Dalmon (2010) enfatizam que a possibilidade de interagir com objetos geométricos constitui um dos principais aspectos a serem contemplados no ensino de Geometria. Segundo os autores, faz-se essencial também oferecer materiais de apoio que deem suporte às atividades conduzidas através da manipulação de objetos, de modo a facilitar o reconhecimento das propriedades geométricas inerentes aos elementos manipulados.

O incentivo ao desenvolvimento de estratégias pedagógicas pautadas na adoção de Objetos de Aprendizagem interativos tais como as propostas recém apresentadas, requer a realização de capacitações docentes sobre ferramentas que possibilitem a produção de tais recursos. Neste contexto, a presente pesquisa dedicou-se à

\footnotetext{
${ }^{2}$ Disponível em: http://www.cinderella.de/
} 
concretização de uma proposta de formação de professores de Matemática para o uso do software GeoGebra ${ }^{3}$, a qual será apresentada na seção seguinte.

\section{Metodologia}

No segundo semestre do ano de 2013, foi proposto e desenvolvido um curso de extensão, na Pontifícia Universidade Católica do Rio Grande do Sul, denominado "Explorando o uso do Software GeoGebra no Ensino da Matemática". O curso tinha como público-alvo professores atuantes nos mais diversos níveis de ensino, bem como alunos de Licenciatura em Matemática, futuros professores desta disciplina.

Com caráter semipresencial, contou-se com 3 encontros aos sábados, totalizando 12 horas, mais um total de 8 horas de tarefas a serem realizadas a distância. Durante o desenvolvimento do curso, participaram 14 alunos, entre os quais alunos de graduação, de mestrado e professores já atuantes na Educação Básica. Dada a heterogeneidade da turma, em um primeiro momento optou-se por usar as ideias propostas por Lieban e Müller (2012), isto é, o início do curso foi dedicado a uma exploração da ferramenta e suas potencialidades, desde as mais simples até algumas mais elaboradas. Foram também apresentados, como motivação, alguns trabalhos já realizados por estes autores e seus alunos, disponíveis em https://sites.google.com/site/geogebrando/ e mostrados na Figura 2 a seguir

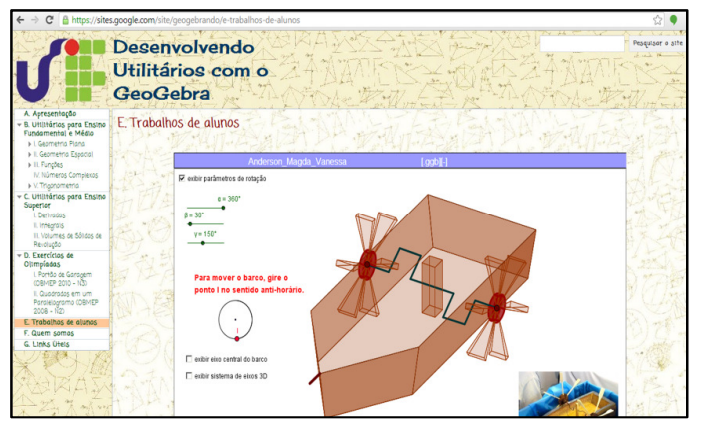

Figura 2- Site Desenvolvendo Utilitários com o GeoGebra - seção Trabalhos de Alunos
Nesses primeiros momentos, após a exploração do software, foram discutidas algumas das construções contidas neste site e possibilidades de exploração destas em sala de aula. Foram sugeridas atividades a serem realizadas com alunos e construções a serem feitas pelos professores, de acordo com seus objetivos em cada situação. Entre os assuntos abordados, encontram-se geometria plana, números complexos, funções e trigonometria.

Como atividade prática, foi solicitado aos alunos que, além das construções realizadas em aula, seguissem os passos recomendados e fizessem algumas construções fora do horário de aula, conforme o arquivo recebido com o passo a passo de cada construção solicitada. Na aula posterior, as dificuldades eram levantadas e possíveis problemas resolvidos de forma conjunta, fazendo com que todos tivessem sucesso na construção de seus OAs.

Como tarefa final, foi solicitado que cada aluno selecionasse um tópico de seu interesse, a partir de uma reflexão sobre as dificuldades que detecta em seus alunos (ou até mesmo colegas) e construísse algum OA com o GeoGebra que pudesse auxiliar estes alunos no aprendizado do conteúdo em questão, seja por meio da experimentação ou simplesmente por visualização, que são as principais características do uso de um software de geometria dinâmica no ensino da Matemática. De fato, a partir de uma

\footnotetext{
${ }^{3}$ Geogebra é um software de Geometria Dinâmica, baseado em código livre, que combina geometria, álgebra, tabelas, gráficos, estatística e cálculo em um único sistema. Disponível em: http://www.geogebra.org/
} 
ferramenta como o GeoGebra, é possível a criação de variadas situações que não seriam possíveis com lápis e papel ou mesmo no quadro, e a promoção da experimentação, isto é, a possibilidade de testar, errar e refazer, de movimentar um objeto e fazer conjecturas é muito importante e facilita a compreensão de conceitos e propriedades. Por outro lado, ainda que a experimentação não seja possível em algumas situações, a simples visualização já pode ser muito mais efetiva a partir da utilização do software.

Dada esta tarefa, para o último encontro foi marcado um momento de apresentações, na qual os objetos criados seriam apresentados aos colegas, bem como discutidas suas possibilidades de utilização.

Encerrando o curso, foi explorada a construção de um Google Sites, o qual pode ser criado por qualquer usuário que possua uma conta no Gmail e se apresenta como um grande aliado do professor na disponibilização de OAs criados com o GeoGebra, uma vez que possui um Gadget previamente programado para que as construções feitas com o software possam ser inseridas no site de forma muito simples, mantendo sua interatividade.

Tais propostas foram ancoradas nas ideias apresentadas na seção 2, especialmente na questão do desenvolvimento de habilidades técnicas, no sentido de que se acredita na importância de o professor aprender a construir seus próprios materiais e disponibilizá-los a seus alunos da forma mais acessível possível para ambos.

\section{Resultados}

Nas seções que seguem, serão apresentados alguns dos resultados obtidos a partir das atividades propostas na Metodologia, bem como as possibilidades e limitações observadas.

\subsection{Produções dos participantes com a ferramenta}

Conforme mencionado na seção 5, no último dia de curso cada aluno deveria trazer uma produção de sua autoria (possivelmente baseada em algum material já existente, por exemplo, na seção "GeoGebra Tube", do site www.geogebra.org) e apresentar aos colegas.

$\mathrm{Na}$ ocasião, observou-se uma grande diversidade de interesses e construções. Os assuntos abordados foram: funções, geometria, sequências, números irracionais e geometria analítica. Destaca-se também uma proposta de uso do Tangran com GeoGebra e a apresentação feita no Prezi por uma das alunas, o que também é um indício de uso de novas tecnologias no ensino de Matemática e que chamou bastante atenção dos colegas, que a questionaram sobre o funcionamento da ferramenta por eles desconhecida.

Pode-se dizer que as construções mais simples foram realizadas pelos professores em exercício, pensando na aplicabilidade em suas salas de aula e na produção de materiais de estudo pelos próprios alunos. Já os alunos de licenciatura e mestrado, de um modo geral, utilizaram de forma mais ampla ferramentas do GeoGebra que foram exploradas no curso, como animações, seletores, caixas para mostrar/esconder objetos, entre outras. Sendo assim, estes estudantes criaram OAs um pouco mais complexos, que servirão mais de base para o professor utilizar como demonstração e propor atividades direcionadas.

Após uma longa discussão e avaliação conjunta em que todos os participantes deram sugestões e fizeram perguntas a seus colegas, elegeu-se o trabalho que mais 
chamou a atenção de todos, devido ao assunto abordado, ferramentas utilizadas, potencial de utilização, organização das atividades propostas, entre outros aspectos. Tal trabalho, que se encontra na Figura 3 a seguir, refere-se à visualização geométrica dos números irracionais da forma $\sqrt{2}, \sqrt{3}$, etc. A partir de um quadrado de lado 1 , é traçada sua diagonal e mostrado ao aluno que sua medida é $\sqrt{2}$. Segue-se então com a criação de triângulos, formando uma espiral e originando os demais números irracionais envolvidos. A cada etapa, o aluno é questionado sobre o que está acontecendo e levado a deduzir os fatos sobre o surgimento dos novos números.

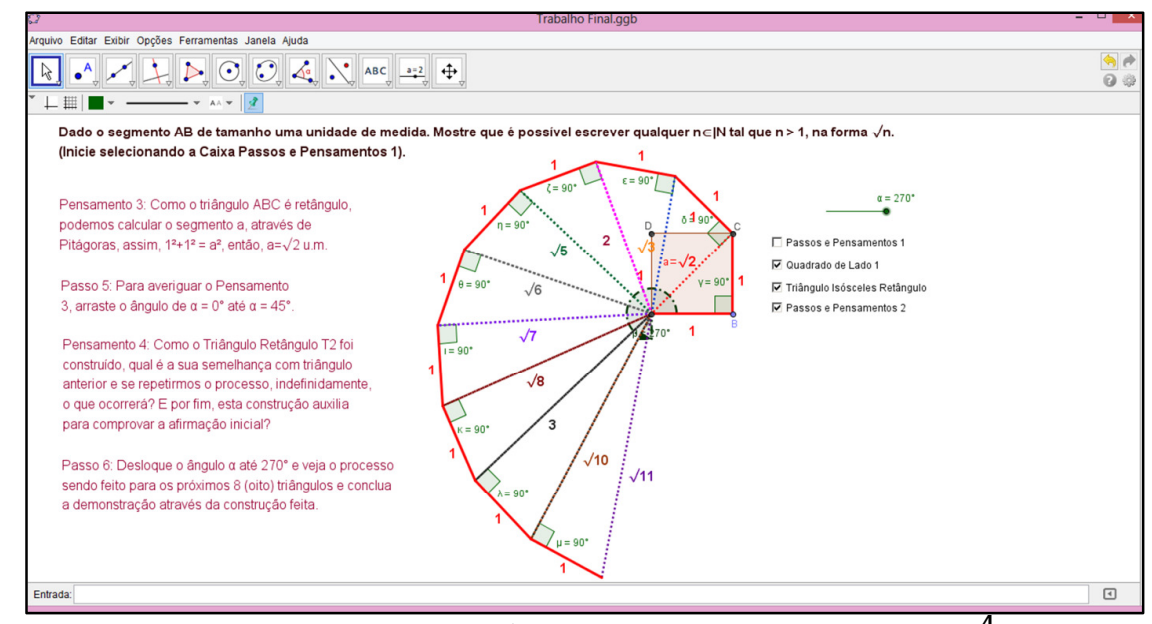

Figura 3 - Estudando números irracionais com GeoGebra ${ }^{4}$

Além disso, cabe ressaltar ainda que as ideias trabalhadas no curso tiveram um amplo alcance também entre alunos da Licenciatura em Matemática que não participaram da atividade de extensão. Como exemplo, tem-se o fato de que dois estudantes que realizaram o curso foram convidados pela professora de uma disciplina de estágio a auxiliar uma colega que estava estagiando em uma escola na cidade de Ivoti-RS em uma aula que ela gostaria de utilizar recursos computacionais com seus alunos para trabalhar funções de $2^{\circ}$ grau. Pensando no que aprenderam, estes dois alunos propuseram uma oficina sobre o tema usando GeoGebra, a qual foi aplicada na referida escola surtindo efeitos positivos em todos os envolvidos.

\subsection{Benefícios e Fragilidades inerentes ao uso da Ferramenta}

A partir da experiência realizada, confirmou-se mais uma vez que o GeoGebra é um software com muitas potencialidades, capaz de abordar diversos assuntos de diferentes maneiras, de acordo com a criatividade do professor. A questão da importância da experimentação também ficou bastante evidente, e observou-se nas construções dos cursistas a preocupação em proporcionar isto a seus alunos.

Com relação ao software em si, existe um grande suporte na rede para usuários, uma vez que o próprio site oficial (www.geogebra.org) contém manuais de utilização, fóruns nos quais interessados de qualquer lugar do mundo podem se comunicar, dar sugestões e resolver dúvidas mutuamente, bem como sugestões de materiais já produzidos na seção GeoGebra Tube.

\footnotetext{
${ }^{4}$ Trabalho desenvolvido pelo aluno Guilherme Rudek Flôres, do curso de Licenciatura em Matemática da PUCRS.
} 
Por outro lado, assim como qualquer outro recurso computacional, o GeoGebra está sujeito a falhas em algumas situações particulares, cabendo ao professor identificálas e, caso não saiba encontrar a solução, ao menos alertar seus alunos para o problema. Tais falhas podem ser relativas a conceitos que o software não identifica, como por exemplo, restrições de domínio de funções. Outra situação, vivenciada no curso, é que existem algumas diferenças entre versões do software, de modo que, se alguma construção é realizada em uma determinada versão e depois o arquivo é aberto em uma versão diferente, alguns comandos podem se perder, comprometendo o funcionamento como um todo. O mesmo problema ocorre na inserção de arquivos no Google Sites, uma vez que o referido Gadget apresenta problemas de reconhecimento de arquivos salvos na versão 4 do GeoGebra em diante.

Apesar das dificuldades relatadas e da possibilidade de o professor, mesmo após uma formação, não ter total domínio sobre a ferramenta, é essencial que este ao menos "se arrisque" a utilizá-la, dados os inúmeros benefícios que esta dinamicidade traz ao aprendizado dos alunos. Conforme mencionam Borba e Penteado (2007) é preciso que o professor saia de sua zona de conforto e entre em uma zona de risco, usando a tecnologia em sala de aula em favor de seus alunos, ainda que não tenha a resposta pronta para todas as situações que poderão vivenciar.

\section{Considerações}

Nos dias atuais, com todas as mudanças que vêm ocorrendo no âmbito educacional e no próprio comportamento dos alunos, o professor de Matemática necessita, além dos conhecimentos específicos de sua disciplina, desenvolver habilidades variadas, de modo especial no uso de recursos tecnológicos, para auxiliar na promoção do aprendizado dos estudantes.

A experiência aqui relatada refere-se a uma busca por instigar em professores e futuros professores de Matemática o desenvolvimento de habilidades referentes à aplicação prática de seus conhecimentos, tal como sugerido por Van Merrienböer e Kirschner (2013). De um modo geral, pode-se dizer que o curso desenvolvido foi bem avaliado pelos participantes, os quais tiveram um aproveitamento satisfatório, haja vista a análise de suas produções.

Com relação à construção do site, comentada na seção 4 , destaca-se que foi feita de forma breve e resumida, porém os participantes relataram que foi interessante vislumbrar a possibilidade de ter um local para armazenar seus arquivos e indicá-los a seus alunos, local este que pode ser construído de forma fácil e rápida.

Sendo assim, espera-se que, como já se teve uma pequena amostra também relatada no texto, as experiências adquiridas pelos participantes e as trocas evidenciadas nesta experiência sejam disseminadas entre outros professores e alunos. Neste sentido, novas edições do curso também deverão ser viabilizadas, novas atividades propostas e avaliações realizadas, com vistas a promover cada vez mais a capacitação de professores para o uso da tecnologia em prol do ensino da Matemática.

\section{Referências}

AGUIRE, Julia M. TURNER, Erin E.; BARTELL, Tonya G.; KALINEC-CRAIG, Crystal; FOOTE, Mary Q.; MCDUFFIE, Amy R.; DRAKE, Corey. Making connections in Practice: How Prospective Elementary Teachers Connect to Children's Mathematical 
Thinking and Community Funds of Knowledge in Mathematics Instruction. Journal of teacher education. v 64, n. 2, p. 178-192, 2012.

BORBA, M. C., PENTEADO, M. G. Informática e Educação Matemática. Belo Horizonte: Autêntica, 2007. (Coleção Tendências em Educação Matemática).

BRANDÃO, Leônidas. O.; ISOTANI, Segi; DALMON, Danilo L. iGeom: Towards an Interactive Geometry Software with Intelligent Guidance Capabilities. Intelligent Tutoring Systems. V. 6095, p 447, 2010.

CHETTY, Raj; FRIEDMAN, John N.; ROCKOFF, Jonah. Measuring The Impacts Of Teachers Ii: Teacher Value-Added And Student Outcomes In Adulthood. National Bureau Of Economic Research. Cambridge: 2013.

DECARO, Marci S.; RITTLE-JOHNSON, Bethany. Exploring mathematics problems prepares children to learn from instruction. Journal of Experimental Child Psychology. v. 113, p. 552-568, 2012.

DEVLIN, Keith. Introduction to Mathematical Thinking. Palo Alto: Keith Devlin, 2012.

HENG, Anne M.; SUDARSHAN, Akhila. "Bigger number means you plus!"Teachers learning to use clinical interviews to understand students' mathematical thinking. Educ Study Math. v. 83, p. 471-485, 2013.

HOFFKAMP, Andrea. The use of interactive visualizations to foster the understanding of concepts of calculus: design principles and empirical results. ZDM Mathematics Education. 43:359-372, 2011.

INEP (Instituto Nacional de Estudos e Pesquisas Educacionais). Disponível em: $<$ http://portal.inep.gov.br/internacional-novo-pisa-opisaeideb $>$. Acesso em: 22 out. 2013.

LIEBAN, D. E.; MÜLLER, T. J. Construção de Utilitários com o Software GeoGebra: uma proposta de divulgação da geometria dinâmica entre professores e alunos. Revista do Instituto GeoGebra Internacional de São Paulo, São Paulo, v.1, n.1, p. 37-50, 2012.

SAEB (Sistema de Avaliação da Educação Brasileira). Disponível em: <http://portal.inep.gov.br/web/saeb/resultados>. Acesso em: 22 out. 2013.

TABAGHI, Shiva G.; SINCLAIR, Nathalie. Using Dynamic Geometry Software to Explore Eigenvectors: The Emergence of Dynamic-SyntheticGeometric Thinking. Technology, Knowledge and Learning. pp. 1-16, 2013.

VAN MERRIËNBOER, Jeroen J. G.; KIRSCHNER, Paul A. Ten Steps to Complex Learning. New York: Routledge, 2013.

WILEY, David. Learning Object Design And Sequencing Theory. Brigham Young University. Dissertation 2000. 